\title{
IMPACT OF TEMPERING PROCESS ON THE YIELD AND COMPOSITION OF QUINOA FLOUR ${ }^{1}$
}

\section{Introduction}

Roller milling is a dry-milling technique that aims to separate the anatomical parts of the seed kernel. Prior to milling, seeds are tempered to facilitate the removal of bran as the plasticity of the bran layer is increased by the absorption of water. Milling and tempering of wheat have been well-studied but these techniques require another approach for quinoa due to its different seed morphology and size.

Roller milling of quinoa is considered a technological challenge but the technique offers the opportunity to separate the different seed tissues. The separation leads to different fractions, rich in different target substances. These fractions can be used as separate food ingredients, which might stimulate the use of quinoa by creating more added value. Nevertheless, studies concerning roller milling and tempering of quinoa are rather limited. This study aims to investigate the impact of different tempering conditions on the roller milling of quinoa.

\section{Material and methods}

Three types of commercially available quinoa seeds (white quinoa: Q1, Q2; red quinoa: Q3) were rollermilled on small scale by a Brabender Quadrumat Junior. Two levels of tempering time ( 8 and $20 \mathrm{~h}$ ) were combined with three levels of tempered moisture $(13,15$ and $17 \%$ ). Flour yield was recorded and the obtained flour was characterised by ash and protein content, and particle size distribution.

\section{Chemical composition}

Higher levels of tempered moisture (15 or $17 \%$ ) were beneficial to reduce the ash content of quinoa flour (Fig. 1a). The ash content of all samples was lower when the quinoa kernels were tempered at $15 \%$ moisture. An additional reduction of flour ash content was observed for 03 when tempering ( 8 and $20 \mathrm{~h}$ ) occurred at $17 \%$ moisture. Tempering at $17 \%$ had no additional effect on the ash content of Q1 or Q2 flours when combined with a tempering time of $8 \mathrm{~h}$. An increase in tempering time, at a fixed moisture level, generally resulted in a higher flour ash content. No effect of tempering time was observed for samples 01 and $Q 3$ if tempered at respectively 15 or $17 \%$, and $17 \%$.

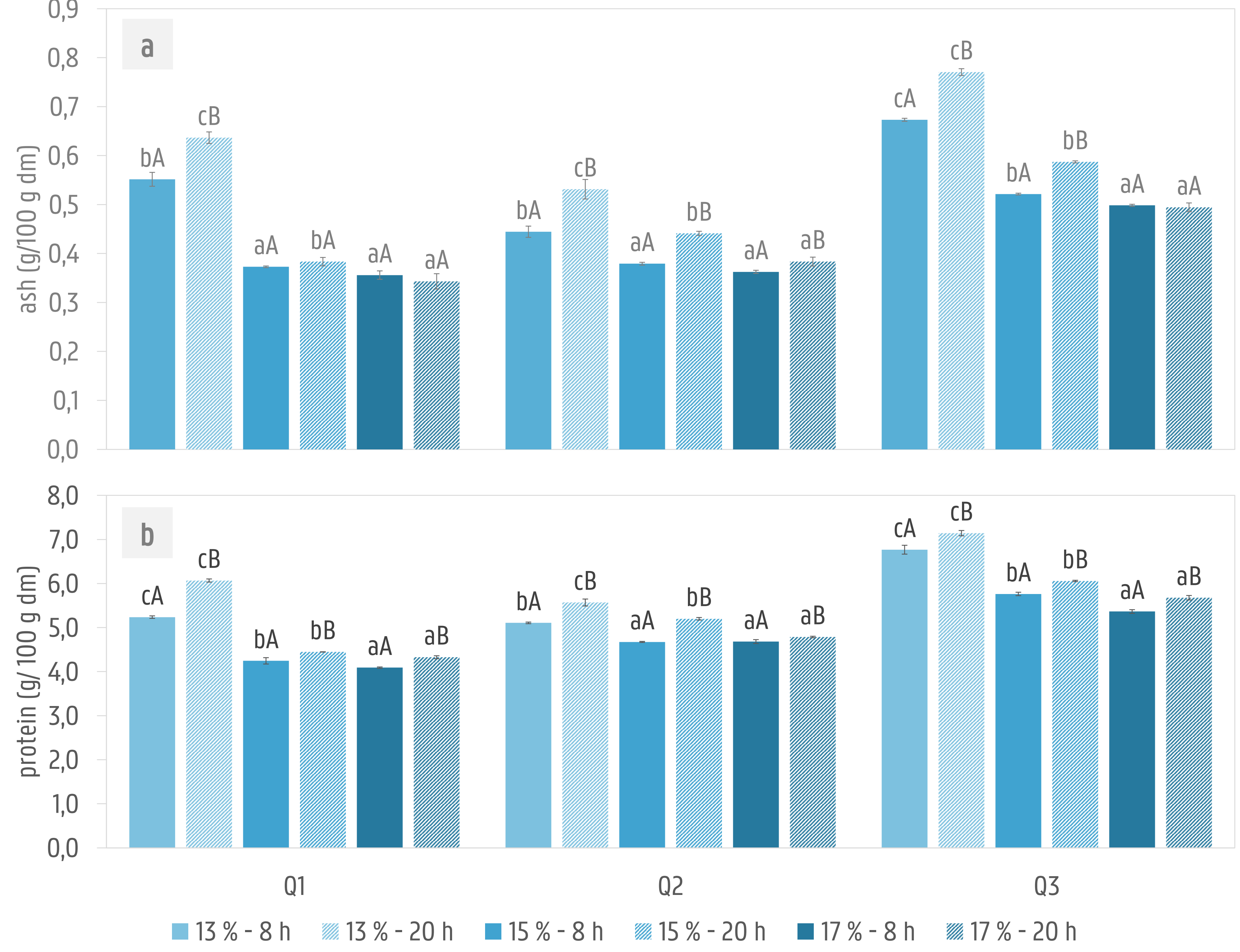

Fig. 1 Ash (a) and protein (b) content ( $\mathrm{g} / 100 \mathrm{~g} \mathrm{dm}$ ) of each quinoa flour (01, 02 and 03 ) per combination of tempering time ( 8 or $20 \mathrm{~h}$ ) and tempered moisture ( 13,15 or $17 \%$ ) ( $n=3)$ ( $A-B$ : effect of tempering time on flour ash/protein content pe sample and level of tempered moisture, a-c: effect of tempered moisture on flour ash/protein content per sample and level of tempering time)

Samples 01 and $Q 3$ showed a significant decrease in flour protein content with every increase in tempered moisture: this was observed for both tempering times (Fig. 1b). Protein content of 02 flours was highest if tempered at the $13 \%$ moisture level. At higher moisture levels (15 and $17 \%$ ) the effect of tempered moisture was time-dependent for sample Q2: no difference in protein content was observed after short tempering $(8 \mathrm{~h}$ ), while long tempering ( $20 \mathrm{~h}$ ) resulted in a higher protein content for moisture level $15 \%$.

Conclusion

The flour milling process of quinoa kernels is mostly influenced by the applied tempered moisture: the flour yield decreases, while the branperisperm separation improves by increasing tempered moisture. Extension of tempering time has no effect on the flour yields of samples Q1 and Q2. The extension, however, tends to reduce the separation efficiency of the milling process as noticed by the increased ash and protein content of the resulting flour. Furthermore, this study suggests that the kernel properties have a distinct impact on the milling behaviour of quinoa: quinoa with a smaller kernel size led to a lower flour yield and flour with a different composition (ash, protein) and particle size distribution. Further research should look into the relationship between kernel properties and roller milling. Despite the differences in kernel properties, the $15 \%$ - $8 \mathrm{~h}$ treatment resulted in an acceptable flour yield for all quinoa samples studied.

De Bock, P., Van Bockstaele, F., Raes, K., Vermeir, P., Van der Meeren, P., \& Eeckhout, M. (2021). Impact of tempering process on yield and composition of quinoa flour. $L W T, 140,110808$.

\section{Flour yield}

mour yields were maximal after tempering at $13 \%$ moisture (Fig. 2). Milling trials indicated that tempered

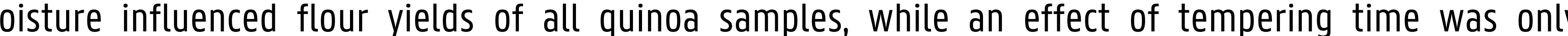
observed for sample 03 . An increase in tempered moisture resulted in a reduction of flour vield. The lowest flour yields were generally obtained for sample Q3; changes in tempering conditions could not increase the flour yield to the same levels of sample Q1 and Q2. The lower milling potential of sample Q3 could be ascribed to the smaller average kernel size, as small-sized kernels are associated with a higher bran to perisperm ratio and a lower potential flour yield.

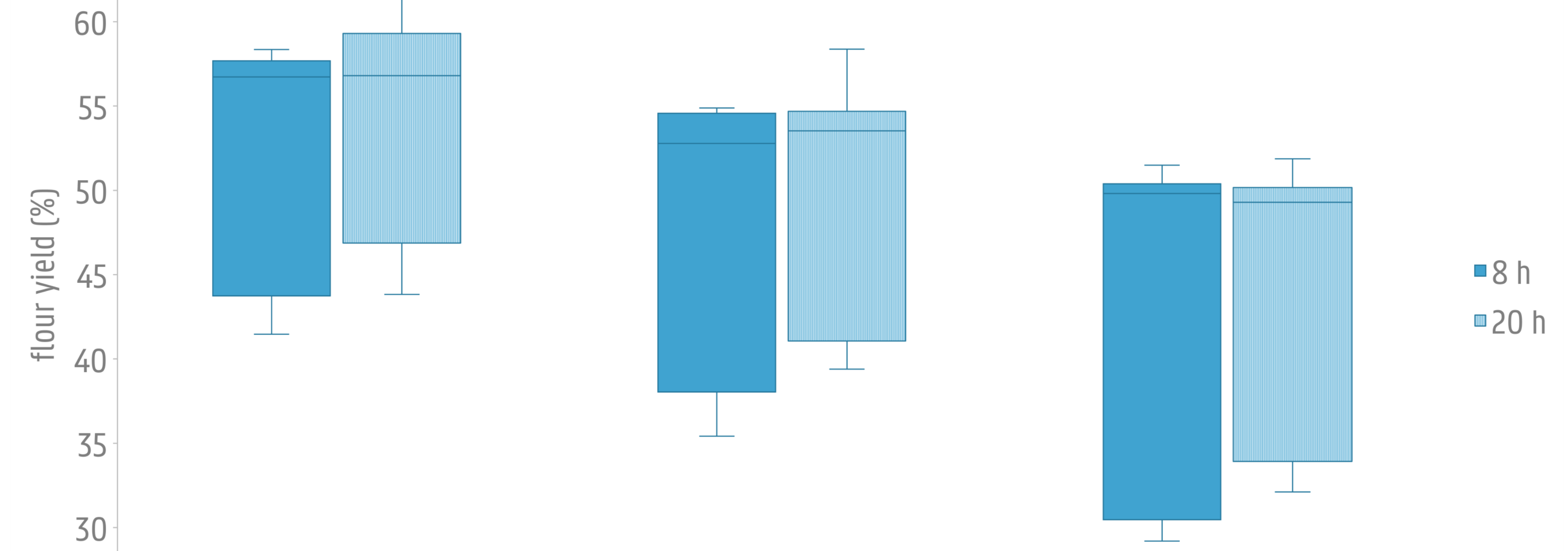

Fig. 2 Flour yield (\%) per combination of tempering time ( 8 or $20 \mathrm{~h}$ ) and tempered moisture $(13,15$ or $17 \%)$

Particle size distribution

For samples Q1 and Q2, an increase in tempered moisture resulted in a reduction of all particle size distribution parameters (Fig. 3a). It is possible that the size of the bran particles sufficiently increased due to the higher tempered moisture and the separation of bran and flour by the sieve (mesh $200 \mu \mathrm{m}$ ) of the laboratory mill therefore improved. The fraction of large particles was considerably higher for Q3 flours (Fig. 3b) as compared to the other samples. This could be related to the small kernel size but could also be ascribed to the higher kernel hardness.

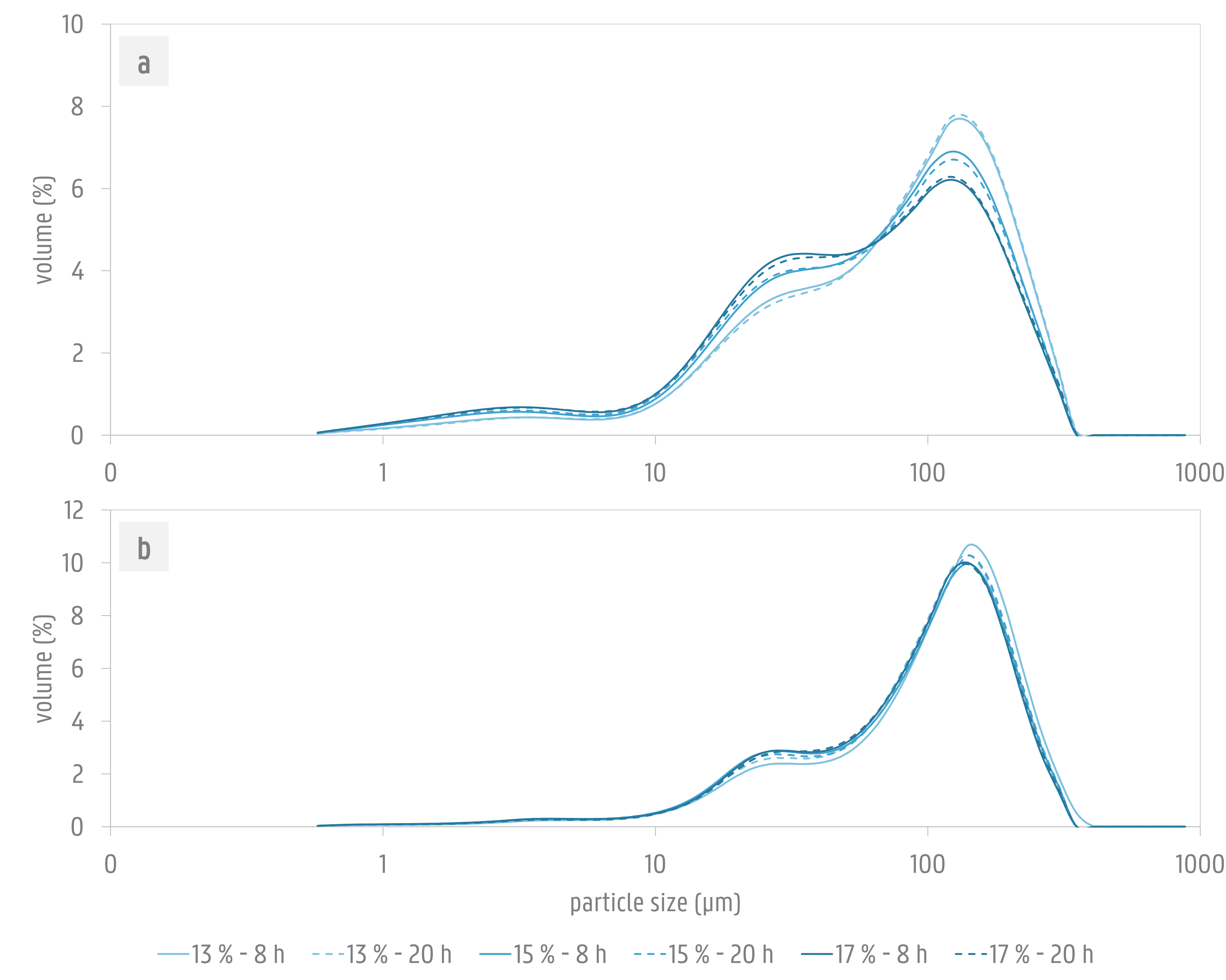

Fig. 3 Particle size distribution of quinoa flour $(\mathrm{a}=01, \mathrm{~b}=03)$ per combination of tempering time $(8$ or $20 \mathrm{~h})$ and tempere moisture $[13,15$ or $17 \%)(n=3)$

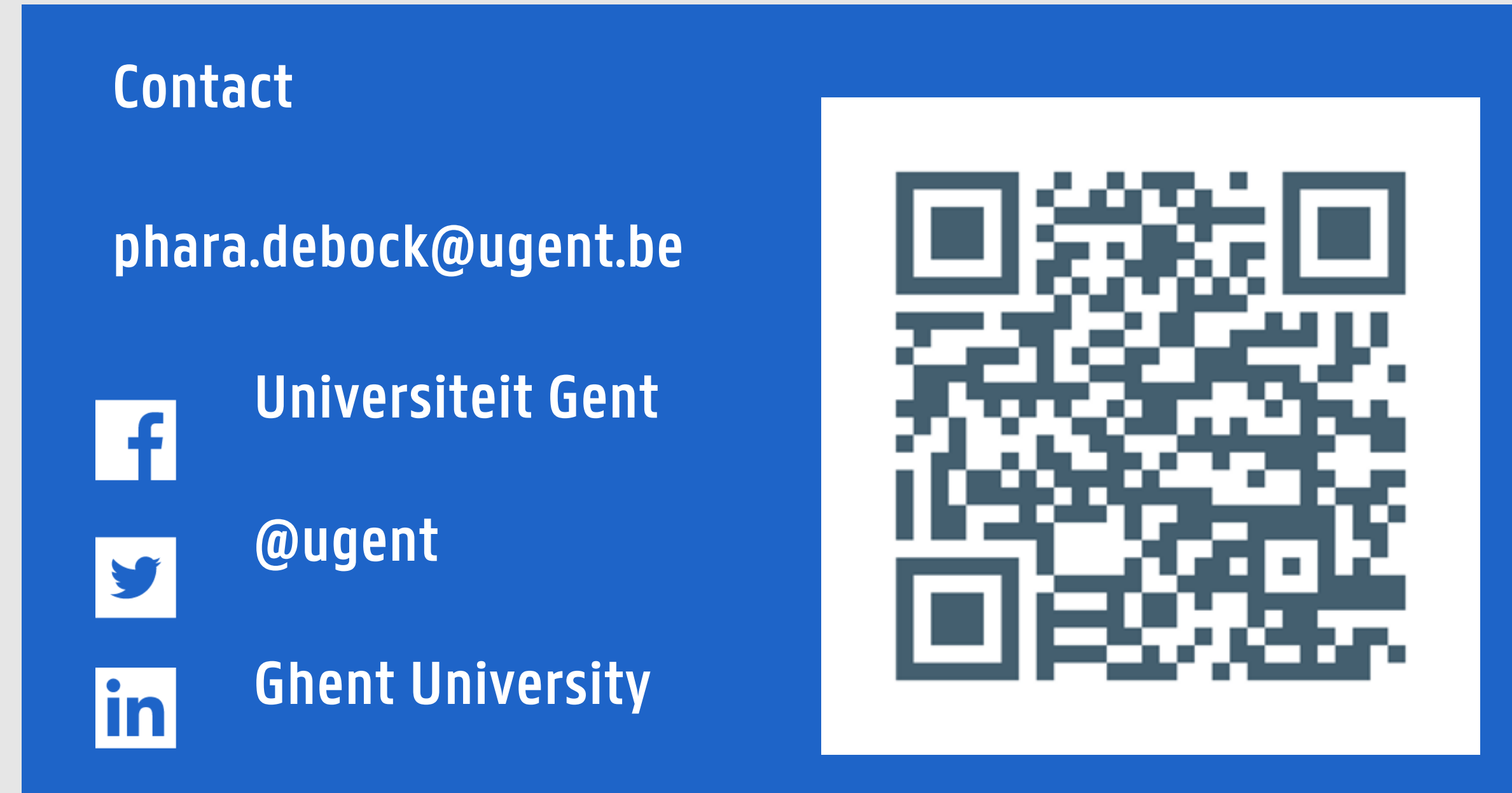

